\title{
In Search of Chaos and Complexity of a Cognitive Language-Learning System
}

\author{
Gaofei Luo ${ }^{1}$ and Sayan Mukherjee $\mathbb{i D}^{2}$ \\ ${ }^{1}$ School of Foreign Languages, Shaoyang University, Shaoyang 422000, China \\ ${ }^{2}$ Department of Mathematics, Sivanath Sastri College, Kolkata, India \\ Correspondence should be addressed to Sayan Mukherjee; msayan80@gmail.com
}

Received 1 July 2020; Revised 7 August 2020; Accepted 14 August 2020; Published 30 September 2020

Academic Editor: Wei Zhou

Copyright (C) 2020 Gaofei Luo and Sayan Mukherjee. This is an open access article distributed under the Creative Commons Attribution License, which permits unrestricted use, distribution, and reproduction in any medium, provided the original work is properly cited.

\begin{abstract}
In this article, we investigate the long-term dynamics of a known cognitive-based language-learning system under the variation of a system parameter. Stability of the equilibrium points is studied. Period root to chaos is investigated by bifurcation analysis. A Lyapunov analysis is performed to verify the complex dynamics in the system. Existence of chaos is confirmed by $0-1$ test. A noiseinduced cognitive phenomenon is proposed under the effect of power noise. Chaotic and nonchaotic dynamics are explored in the noise-induced system. Furthermore, disorder as well as complexity, are investigated for both the systems using the concept of weighted recurrence. The whole analysis can be effective to understand the dynamical features and nonlinear structure of the cognitive language-learning model.
\end{abstract}

\section{Introduction}

Human language is an expressible complex communication system which consists of phonemes, words, phrases, sentences, poetry, and publications [1-4]. Grammar is itself an internal computational system that is used to represent a sentence in the language-learning process [5-7]. People store this internal grammar in the subconscious mind and spontaneously explore it to develop linguistic behaviour. So, language changes over time and hence dynamics is an obvious property of it. Small changes in linguistic data accumulation and interaction with different languages can produce unpredictably complex dynamics in the learning system $[1,8,9]$. As data accumulation and its utilization depend on the human mind, complex dynamics is an effective tool to study the behaviour of cognitive-based language-learning (CBLL) system [10-12]. In [9], it has been already established that chaotic dynamics exist for a CBLL system. However, it does not contain "forgetting" property in the cognitive growth [9]. In this paper, the existence of deterministic and noise-induced chaos [13-19] in a known
CBLL system was investigated for the first time. Moreover, the complexity of the respective dynamics has been studied which is not explored indeed.

Chaos can be verified by measuring the exponential divergence between the phase space trajectories [14]. Lyapunov exponent (LE) is one of the powerful tools that can measure the exponential divergence properly [20, 21]. An alternative measure $0-1$ test method [22, 23] was also proposed that can successfully characterize regular as well as chaotic behaviour of the system [22, 23]. Moreover, it is applicable for both deterministic and noise-induced system [24]. The invariant characteristic of phase space can be characterized by measuring the complexity of the corresponding system [15]. It can identify the disorder in the phase space trajectories by utilizing the concept of Shannon entropy [25]. Several entropy measures have been proposed to quantify the complexity [26-37]. Recurrence-based entropy is one of the effective measures that can be applied in any dimensional system [38, 39]. However, it does not correlate with the proper dynamics of the system due to the selection of incorrect threshold [39]. To overcome this 
situation, a new entropy measure has been proposed based on weighted recurrence plot [40].

This manuscript is organized as follows. In Section 2, a cognitive language-learning system is considered and illustrated by the schematic diagram. Complex dynamics is investigated for both the CBLL and its noise-induced system in Sections 3.1 and 3.2, respectively. In section 3.1, equilibrium points and the respective stability both are determined, and multiperiodicity and chaos are investigated by bifurcation analysis, Lyapunov study, and 0-1 test method. In Section 3.2, a noise-induced CBLL phenomenon is proposed and described by a schematic representation. Furthermore, chaos in the noise-induced CBLL is also investigated numerically by the $0-1$ test method in the same section. The corresponding phase space behaviour is also investigated for the CBLL and its noise-induced systems in Sections 3.1 and 3.2, respectively. Section 4 discusses the complexity of both the systems using weighted recurrence entropy analysis. Section 5 is the conclusion.

\section{Mathematical Model of a Cognition-Based Language Learning (CBLL) Phenomenon}

We consider a cognitive language growth model proposed in [9]. In this model, the growth in language is subject to the constraint of forgetting or loss of proficiency in the human brain. A schematic diagram is given in Figure 1 to describe the CBLL model.

As cognitive learning is always dependent on some resource [9], we mentioned a set of language resources in Figure 1. The human brain collects the information from language resource and then processes it for learning. So, resource growth factor is one of the major parameters in cognitive learning. In Figure 1, $r^{\prime}$ denotes the resource growth parameter. Furthermore, our brain is not capable to store all information with stabilized condition. Thus, forgetting or loss of information affects the learning process. In Figure 1, $m^{\prime}$ denotes the forgetting parameters which have been incorporated with the CBLL system in [9]. Mathematically, the system can be expressed by $L_{i+1}=$ $f\left(L_{i}, r^{\prime}, m^{\prime}\right)$, where $L_{i}$ and $L_{i+1}$ represent input and output, respectively. In [9], the explicit form of the $f$ is given by $f\left(L_{i}, r^{\prime}, m^{\prime}\right)=\left(1+r^{\prime}-m^{\prime}\right) L_{i}$. According to the study in [9], $r^{\prime}$ depends on intrinsic growth rate $r$, carrying capacity $K$, and also the input $L_{i}$ by $r^{\prime}=r\left(K-L_{i} / K\right)$. Furthermore, it has been also established in [9] that the forgetting parameter $m^{\prime}$ depends on intrinsic forgetting growth $m, K$, and $L_{i}$ by $m^{\prime}=m\left(L_{i} / K\right)$. Thus, the CBLL system is given by

$$
L_{i+1}=(1+r) L_{i}-\left(a+\frac{m}{K}\right) L_{i}^{2}, \quad m<K,
$$

where $a$ is known as braking parameter given by $a=r / K$. For our purpose, we fix the respective values of $a, m$, and $K$ by $0.01,0.1$, and 0.5 , respectively. By solving (1), we get an output which is called language growth oscillation or simply language growth of the system. In Figure 1, the language growth is shown at the extreme right panel.

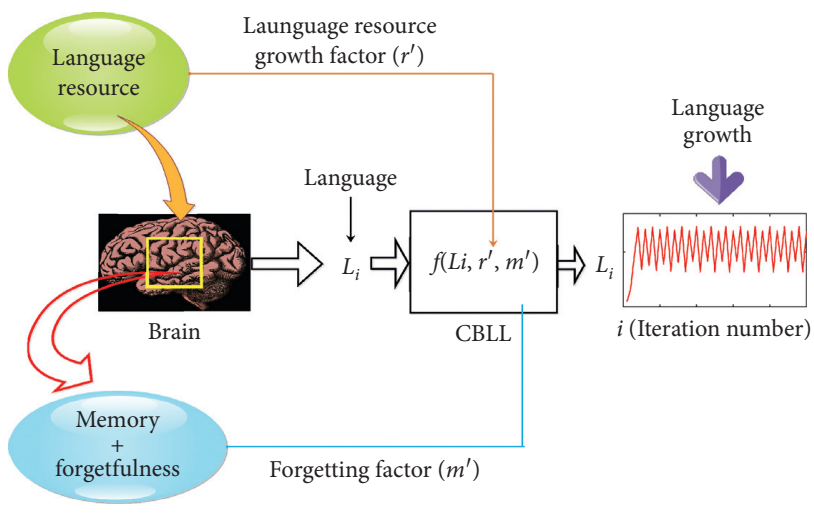

FIgURE 1: Schematic diagram for CBLL Phenomenon.

\section{Dynamics of the CBLL System}

3.1. Nonnoisy Condition. We first investigated equilibrium points of (1) and the respective stabilities under the variation of $r$. To calculate equilibrium points of (1), we consider an equation: $L=(1+r) L-(a+(m / K)) L^{2}$, where $L$ represents equilibrium point in (1). The equation gives two solution of $L$, say $L_{1}$ and $L_{2}$ given by $L_{1}=0$ and $L_{2}=r /(a+(m / K))$. As we fixed $a=0.01, m=0.1$, and $K=0.5, L_{2}$ can be considered as $L_{2}=\eta r$, where $\eta=1 /(a+(m / K))=$ constant. Then, stability of $L_{1}$ and $L_{2}$ are investigated by perturbing system (1) with $\delta L_{i}=L_{i}-L$ (perturbation). Then, by Taylor's theorem, we can have $\delta L_{i+1}=(\mathrm{d} f(L) / \mathrm{d} l) \delta L_{i}$ with the small perturbation. Hence, $\left|\delta L_{i+1} / \delta L_{i}\right|=|\mathrm{d} f(L) / \mathrm{d} l|$. It indicates that $\delta L_{i+1}<\delta L_{i}$ if $|\mathrm{d} f(L) / \mathrm{d} l|<1$, i.e., the system is said to be stable and unstable if $|\mathrm{d} f(L) / \mathrm{d} l|<1$ and $>1$, respectively. We thus investigate stability region of $L_{1}$ and $L_{2}$ by verifying $|\mathrm{d} f(L) / \mathrm{d} l|<1$ with the variation in $r$. As $(\mathrm{d} f(L) / \mathrm{d} l)=1+r$ at the equilibrium points $L_{1}=0$, we get $(\mathrm{d} f(L) / \mathrm{d} l) \geq 1$ for $r \geq 0$. As $(\mathrm{d} f(L) / \mathrm{d} l)=1$ for $r=0$, no conclusion can be inferred. Since $(\mathrm{d} f(L) / \mathrm{d} l)>1$ for all $r>0$, it implies $L_{1}$ is unstable in nature. Same analysis is done for $L_{2}=\eta r$. The analysis shows $|(\mathrm{d} f(L) / \mathrm{d} l)|<1$ for $0<r<2$. The stability regions for $L_{1}$ and $L_{2}$ are given in Figure 2.

We next investigate bifurcation phenomena of (1) for $r \in[1.5,3]$. The corresponding graph is shown in Figure 3 .

We divide the whole graph into three windows. From the first window, it can be observed that system (1) produces single and double periods for $r \in[1.5,2.45]$, whether the second window shows the existence of two as well as four periods in (1) for $r \in(2.45,2.55]$. On the other hand, multiple periods can be seen for the same system with $r \in(2.55,3]$ (see the third window in Figure 3). So, the entire bifurcation analysis indicates that the dynamics of (1) may be complex with the increase of $r \in[1.5,3]$. To confirm this, we have investigated Lyapunov exponent (LE) for the system (1) with $r \in[1.5,3]$. The LE is calculated by

$$
\lambda=\lim _{n \longrightarrow \infty} \frac{1}{n} \sum_{i=1}^{n} \log \left|\frac{\mathrm{d} f\left(L_{i}\right)}{\mathrm{d} L_{i}}\right|,
$$

where $n$ represents length of the $\left\{L_{i}\right\}$.

Figure 4(a) shows fluctuation of $\lambda$ over $r \in[1.5,3]$. It can be observed from Figure 4(a) that the oscillation of $\lambda$ always 


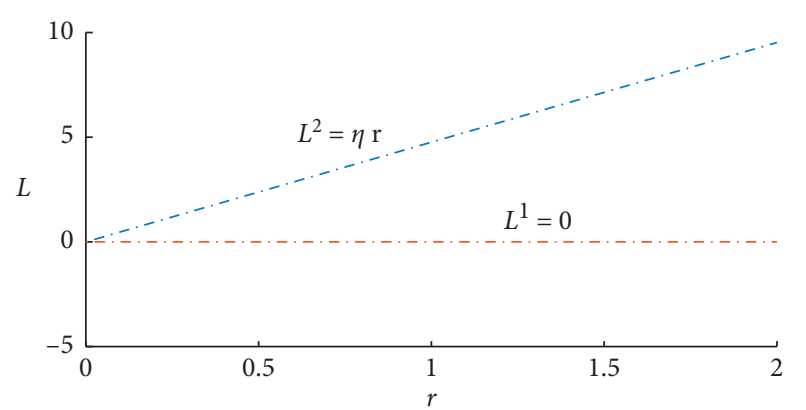

FIgURE 2: $L$ vs. $r$ plot represents regions of stability of the two fixed points, $L_{1}$ and $L_{2}$, of system (1).

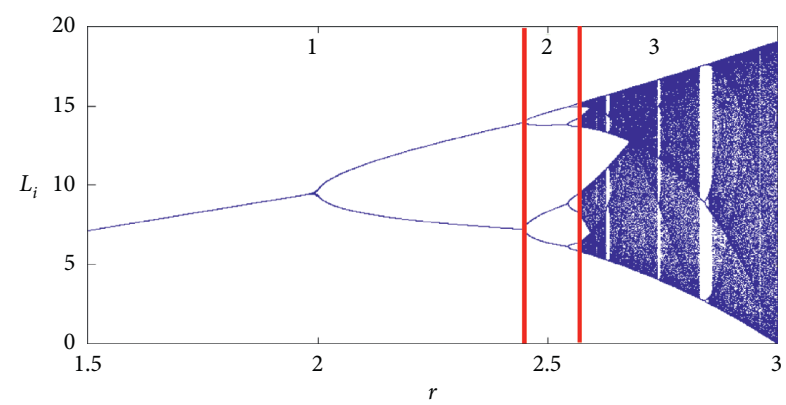

FIgURE 3: $L_{i}$ vs. $r$ bifurcation diagram of system (1) with the initial condition $L_{1}=1$ and $r \in[1.5,3]$. For each $r$, the roots are calculated by solving (1) with length $N=500000$. Here, 1,2 , and 3 indicate number of successive windows for $r$ defined by [1.5, 2.45], $(2.45,2.55],(2.55,3]$, respectively.

fluctuates in $[-4,0]$ for $r \in[1.5,2.55]$. It assures nonchaotic dynamics in (1) over $r \in[1.5,2.55]$. Furthermore, $\lambda<0,>0$ and $=0$ can be seen for $r>2.55$. It indicates chaotic as well as nonchaotic dynamics exists in (1) for $r \in(2.55,3]$. However, it can be investigated that the values of positive $\lambda \approx 0$ for some $r \in(2.55,3]$ in Figure 4(a). In this case, chaos cannot be confirmed for the system as two different trajectories show very small divergence with the perturbation in initial condition. We thus investigate chaos in (1) by $0-1$ test method. In this method, a solution component, say $\{x(n)\}_{k=1}^{N}$ (N being the length of the component) of a system is considered [22,23]. Then, $\{x(n)\}_{k=1}^{N}$ decomposes into two components by the transformation:

$$
\begin{aligned}
& p(n, c)=\sum_{j=1}^{n} x(j) \cos (j c), \\
& q(n, c)=\sum_{j=1}^{n} x(j) \sin (j c), \quad c \in(0, \pi) .
\end{aligned}
$$

The chaotic and nonchaotic dynamics of a system can be identified by the nature of $(p, q)$-plots. In fact, regular and Brownian motion-like structure of the $(p, q)$-plots corresponds with respective nonchaotic and chaotic dynamics of the system $[22,23]$. We have investigated $(p, q)$-plots for system (1) with $r \in[1.5,3]$. Figures $4(\mathrm{~b})$ and $4(\mathrm{c})$ show two $(p, q)$-plots for $r=2.5$ and 3 , respectively.

From Figure $4(\mathrm{~b})$, it can be seen that the $(p, q)$-plot contains a regular geometrical structure. It indicates nonchaotic dynamics in (1). On the contrary, Brownian motion-like structure can be observed in Figure 4(c). It corresponds to chaotic dynamics in (1). To quantify chaotic and nonchaotic dynamics, we compute fluctuation of $K_{c}(r),(r \in[1.5,3])$ given by

$$
K_{c}=\lim _{n \longrightarrow \infty} \frac{\log M_{c}(n)}{\log n},
$$

where $M_{c}(n)$ is defined as

$$
\begin{aligned}
M_{c}(n)= & \lim _{N \longrightarrow \infty} \frac{1}{N} \sum_{j=1}^{N} p_{c}\left[(j+n)-p_{c}(j)\right]^{2} \\
& +\left[q_{c}(j+n)-q_{c}(j)\right] .
\end{aligned}
$$

The values of $K_{c} \approx 0$ and 1 correspond nonchaotic and chaotic dynamics of the system. Figure 4(d) shows fluctuation of $K_{c}$ over $r \in[1.5,3]$. From Figure 4(d), it can be observed that values of $K_{c} \approx 0$ for all $r \in[1.5,2.55]$. It assures nonchaotic dynamics in (1). It can be also observed that, $K_{c} \approx 1,0$ and $K_{c} \approx 1$ may happens for the same system with $r \in(2.55,3]$. It implies chaotic as well as nonchaotic dynamics exists in (1) for $r \in(2.55,3]$.

We further investigated oscillation of $L_{i}$ and its corresponding phase space behaviour of system (1) over $r \in(1.5,3]$. Figures 5(a) and 5(c) show oscillation of $L_{i}$ for $r=2.5$ and 3 , respectively. It can be observed from the figures that variation in $L_{i}$ for $r=2.5$ is lesser than that for $r=3$. It indicates system (1) reveals more complex language growth for $r=3$ compared to the same for $r=2.5$. Thus, nature of language growth can be investigated in (1) for the entire range of $r$. However, long-term dynamics in (1) cannot be possible to predict by investigating $L_{i}$ only. So, a phase space analysis is carried out for (1) with $r \in[1.5,3]$. In order to investigate phase space of (1), we construct Poincare' plot for each $r$. Figures 5(b) and 5(d) represent two Poincare' plots with $r=2.5,3$ respectively. In 5(b), the corresponding plot shows few cluster of $\left(L_{i}, L_{i+1}\right)$ clouds. As maximum number of $\left(L_{i}, L_{i+1}\right)$ points coverages to each another, it indicates stable dynamics in (1) for $r=2.5$. On the contrary, the Poincare plot in Figure 5(d) indicates the well-known chaotic logistic curve for $r=3$. It implies a strong correlation between the phase space and 0-1 test analysis. Moreover, existence of this correlation can also be verified for all $r \in[1.5,3]$. In this way, existence of chaotic as well as nonchaotic dynamics in (1) is identified.

3.2. Noisy Condition. To study the dynamics of the CBLL system under the effect of noise, we incorporate a multiplicative noise $\xi$ in (1) with the noise strength $c$. The whole phenomenon is described in a schematic diagram given by Figure 6 . In Figure 6, (.) indicates multiplication. The green arrow denotes the incorporation of $\xi$ with the intrinsic parameter $r$. The functional form of the resultant CBLL system is given by $f\left(L_{i}, r \cdot c \xi, m\right.$ ) (see Figure 6). 


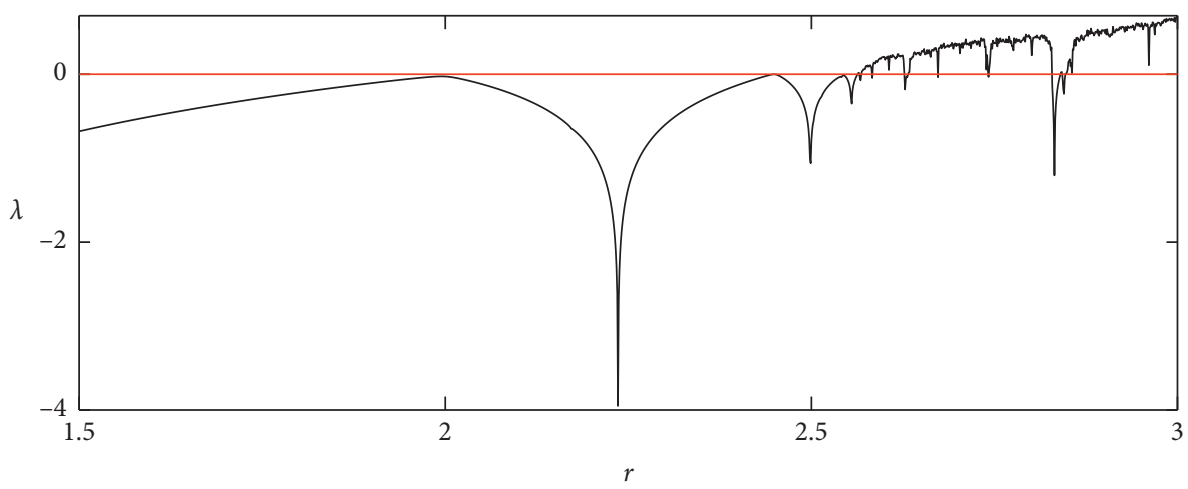

(a)

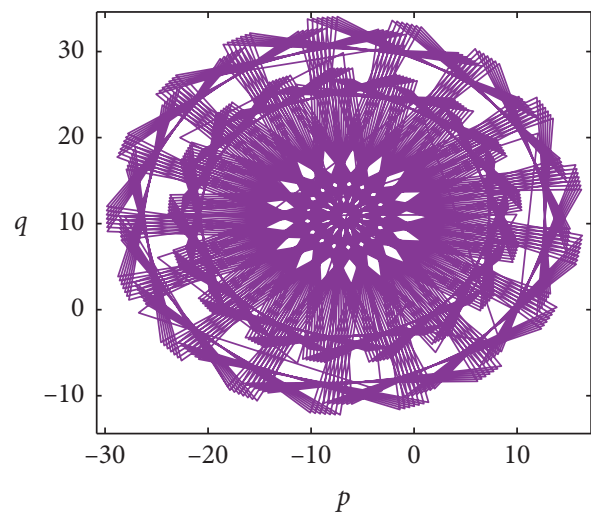

(b)

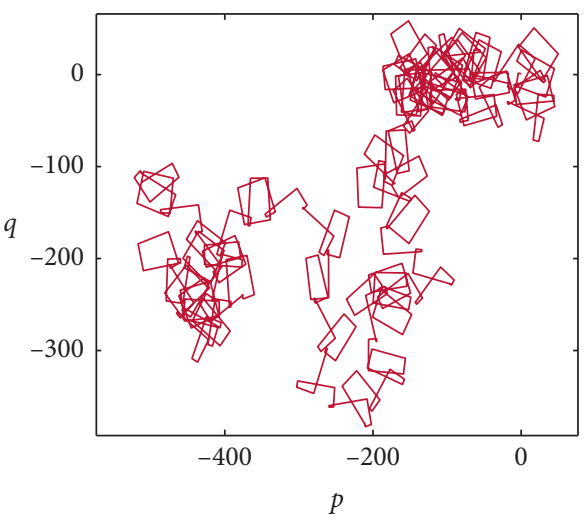

(c)

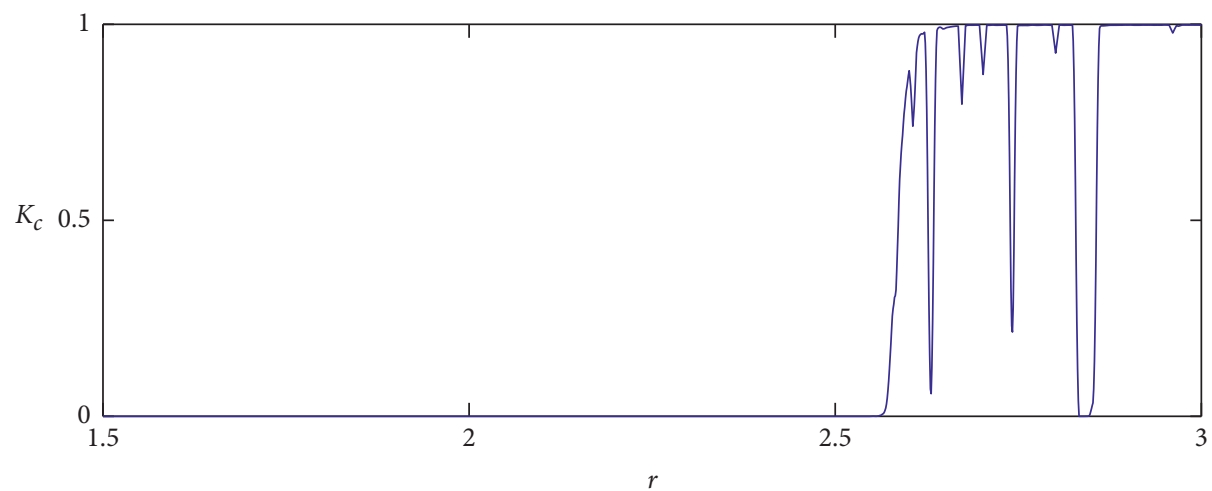

(d)

Figure 4: (a) $\lambda$ vs. $r$ graph of system (1) for $r \in[1.5,3]$. (b, c) $(p, q)$-plots of (1) with $r=2.5$ and 3, respectively. To calculate $p$ and $q$, we have considered $c=(2 \pi / 3)$. (d) $K_{c}$ vs. $r \in[1.5,3]$ graph of the same system. In each case, $K_{c}$ is calculated with $n_{\text {cut }}=(N / 10)$.

The noise-induced system is thus given by

$$
L_{i+1}=(1+r \cdot c \xi) L_{i}-\left(a+\frac{m}{K}\right) L_{i}^{2}, \quad m<K,
$$

where $r, K$, and $a$ represent the same as mentioned for the nonnoisy case. For our purpose, $\xi$ is taken as $1 / f^{\beta}$-noise with $\beta=1$.

In this case, we have investigated chaotic and nonchaotic dynamics in (6) under both the variation of $r \in[1.5,3]$ and $c \in[0.1,1]$, respectively. To do so, we have applied the $0-1$ test method in (6). Figures $7(\mathrm{a})$ and $7(\mathrm{~b})$ show respective $(p, q)$-plots for $r=1.5$ and $r=3$ with the fixed $c=0.5$. From the respective $(p, q)$ plots, a regular geometric structure and
Brownian motion-like movement can be observed for $r=$ $1.5, c=0.5$ and $r=3, c=0.5$, respectively. It indicates nonchaotic and chaotic dynamics in (6), respectively. These can be also identified for all $r \in[1.5,3]$ and $c \in[0.1,1]$.

In the next, fluctuation of $K_{c}$ is also measured for the same $r \in[1.5,3]$ with $c=0.5$. The corresponding graph is given in Figure 7(c). It can be observed from Figure 7(c) that $K_{c} \approx 1$ for all $r \geq 2.4$ and $K_{c} \approx 1$ for $r<2$.4. It assures chaotic and nonchaotic dynamics in (6) for $r \geq 2.4$ and $r<2.4$, respectively. Hence, the method of $0-1$ test can classify chaotic as well as nonchaotic phenomena in (6) over $r \in[1.5,3]$ with fixed $c=0.5$. Furthermore, fluctuation of $K_{c}$ is investigated over $(r, c) \in[1.5,3] \times[0.1,1]$. Figure $7(\mathrm{~d})$ shows the 


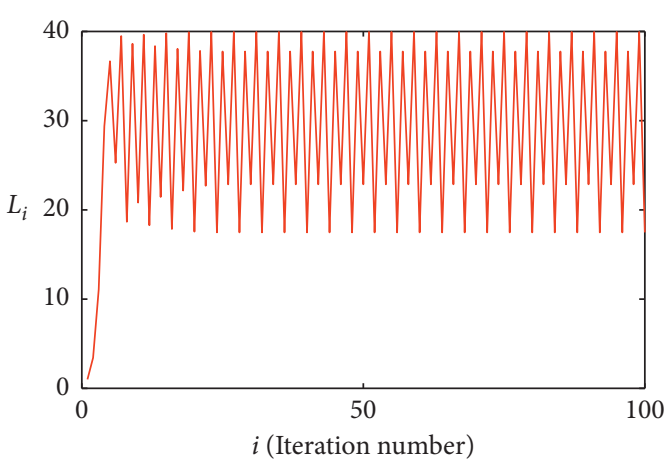

(a)

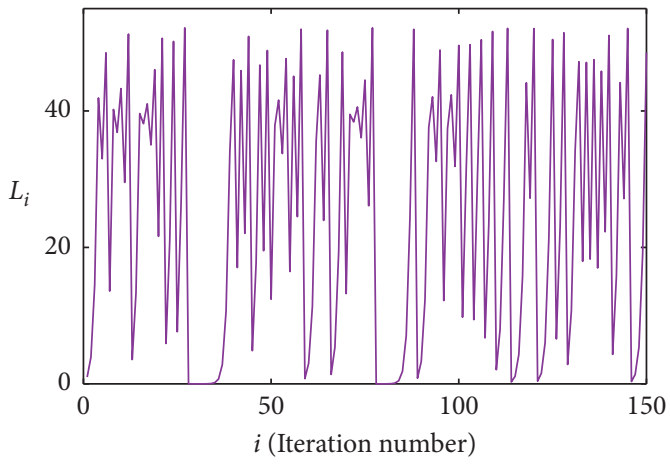

(c)

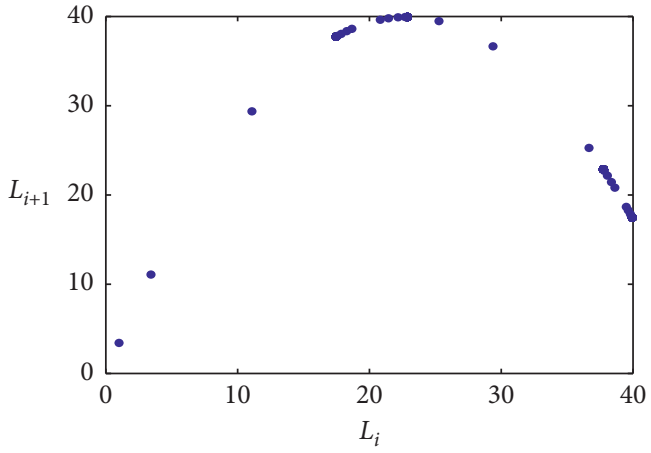

(b)

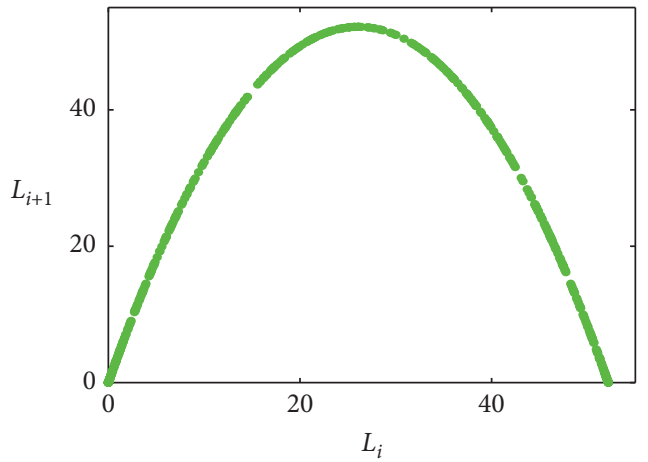

(d)

Figure 5: (a, c) represent $L_{i}$ vs. $i$ graphs with $r=2.5$ and 3, respectively. The corresponding Poincare plots are given in (b, d), respectively. In order to construct the Poincare plot, we have considered last 5000 points of the solution $L_{i}, i=1,2, \ldots, 50000$.

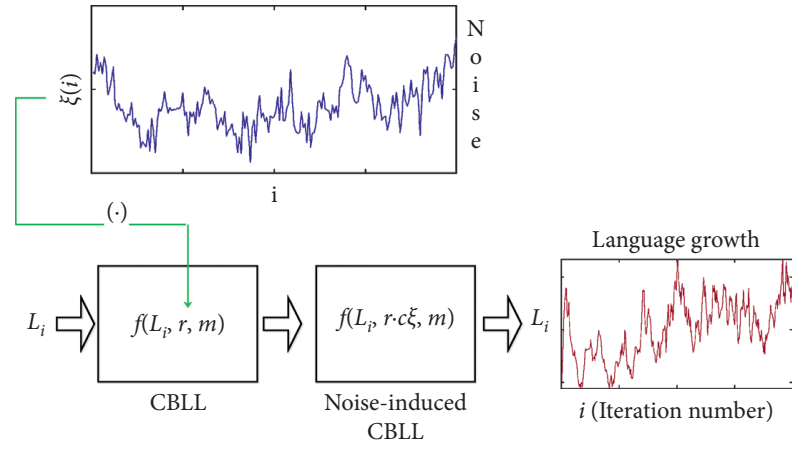

FIGURE 6: Schematic diagram for noise-induced CBLL phenomenon.

corresponding contour diagram. From the figure, it can be seen that yellow, green, blue, pink, and red regions are comparatively smaller than the dark red region. As dark red region corresponds $K_{c} \approx 1$ and other regions correspond $K_{c} \approx 1$, respectively, it implies chaos in (6), which can be observed for almost all $(r, c) \in[1.5,3] \times[0.1,1]$. It can be also observed that $K_{c} \in[0.58,0.9]$ for $r>1.5$ and $c \in[0.1,0.3]$. It follows nonchaotic dynamics in (6) with the increasing $r$ for the noise strength $c \in[0.1,0.3]$. Furthermore, an increasing trend in $K_{c}$ with the increase of $r \in[1.5,3]$ with $c \in(0.3,0.5)$ can be observed for $(6)$. As $K_{c}$ attains its maximum value (i.e., 1) in this case, chaotic dynamics in (6) can be first observed in the region
$[1.5,3] \times(0.3,0.5)$. We call it first chaotic paradigm for system (6). For the remaining region, i.e., $[1.5,3] \times[0.5,1]$, dark red color confirms chaotic dynamics in (6). It establishes the existence of complex dynamics in the noise-induced CBLL system. Moreover, analysis of the 0-1 test method also differentiates the chaotic and nonchaotic paradigm for the same system.

We also investigated oscillation of $L_{i}$ and its corresponding phase space nature of system (6) over $r \in[1.5,3]$ and $c \in[0.1,1]$. Figures $8(\mathrm{a})$ and $8(\mathrm{c})$ show oscillation of $L_{i}$ for $r=1.5(c=0.5)$ and $r=3(c=0.5)$, respectively. It can be observed from Figures 8(a) and 8(b) that abrupt changes in $L_{i}$ for $r=3(c=0.5)$ is higher than that for $r=1.5(c=0.5)$. It indicates, more complex oscillation in $L_{i}$ for $r=3(c=0.5)$ compared to the same for $r=1.5(c=0.5)$. By comparing the $L_{i} s$ in (1) and (6), it can be observed that $L_{i}=0$ for some $i$ in the deterministic chaos whether in the noise-induced chaos, $L_{i}$ does not vanishes for any $i, r \in[1.5,3], c \in[0.5,1]$ (see Figures 5(a) and 5(c) and Figures $8(\mathrm{a})$ and $8(\mathrm{c})$ ). To investigate long-term behaviour of (6), Poincare plots are constructed for the same $r$ and $c$, respectively. Figures $8(\mathrm{~b})$ and $8(\mathrm{~d})$ represent two Poincare plots with $r=1.5(c=0.5)$ and $r=3(c=0.5)$, respectively. It can be seen that the corresponding Poincare cloud for $r=1.5(c=0.5)$ is less distorted compare with the same for + . The oscillation in $L_{i}$ and its corresponding phase space behaviour can also be investigated for $(r, c) \in[1.5,3] \times[0.1,1]$. It gives a strong correlation with the fluctuation in $K_{c}$, as shown in Figure 7(d). 


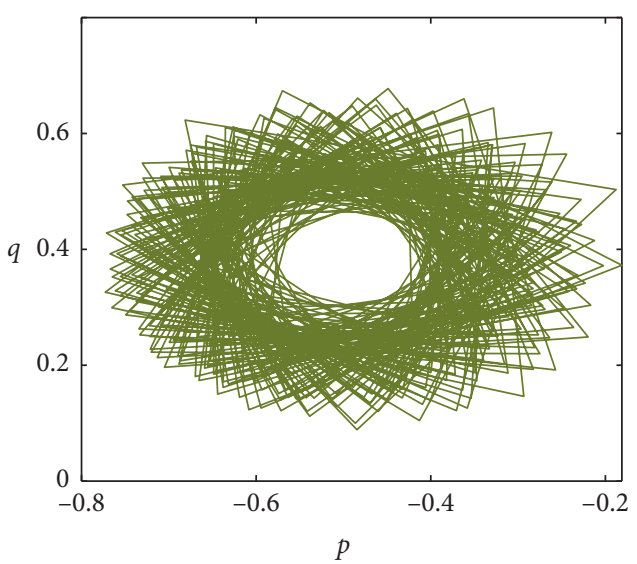

(a)

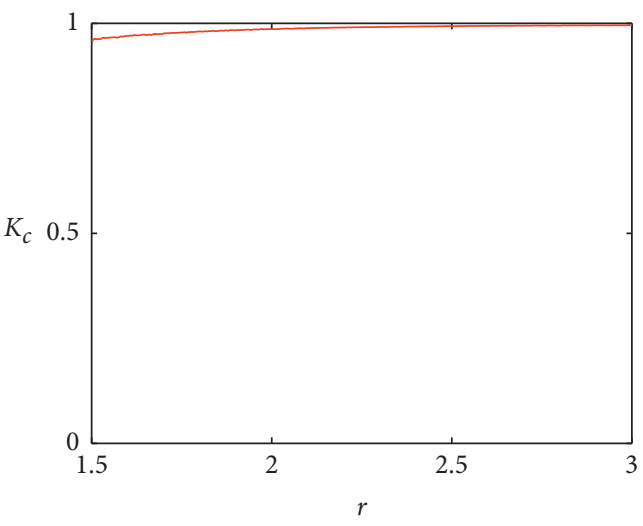

(c)

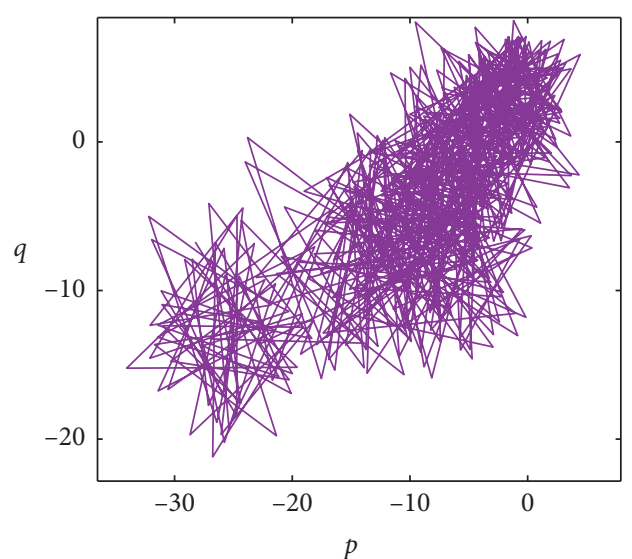

(b)

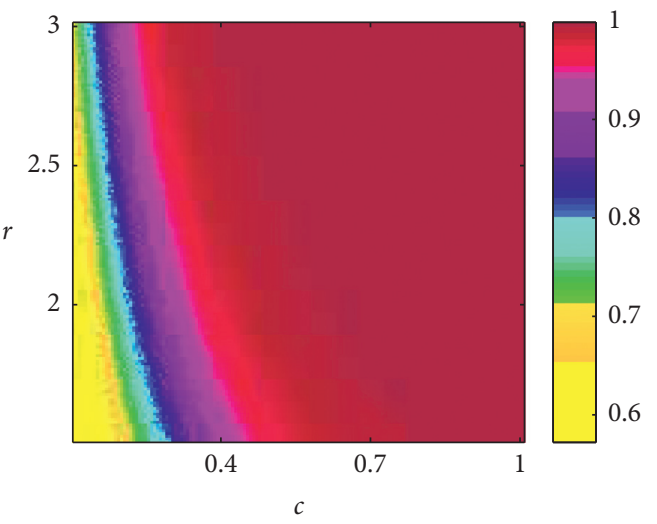

(d)

Figure 7: $(\mathrm{a}, \mathrm{b})(p, q)$-plots of $(6)$ with $r=1.5(c=0.5)$ and $3(c=0.5)$, respectively. To calculate $p$ and $q$, we have considered $c=2 \pi / 3$. $K_{c}$ vs. $r \in[1.5,3](c=0.5)$ graph for $L_{i}$ obtained from (6) given in (c). (d) $K_{c}(r, c)$ matrix plot for $(r, c) \in[1.5,3] \times[0.1,1]$. In each case, $K_{c} s$ are calculated with $n_{\text {cut }}=N / 10$. The color bar indicates values of $K_{c}$.

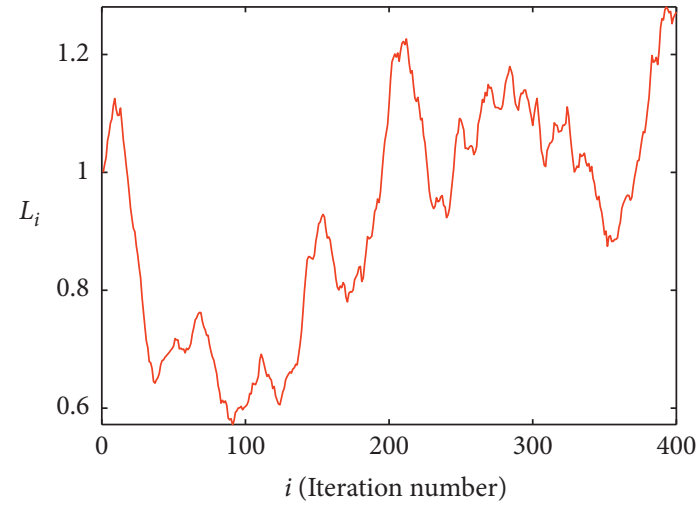

(a)

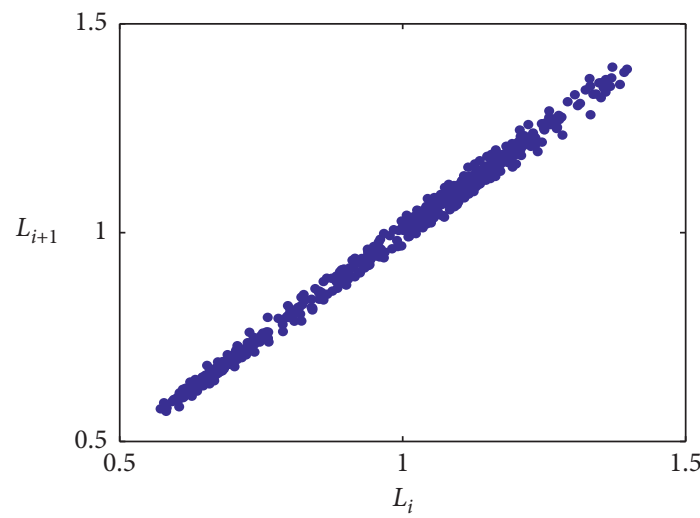

(b)

Figure 8: Continued. 


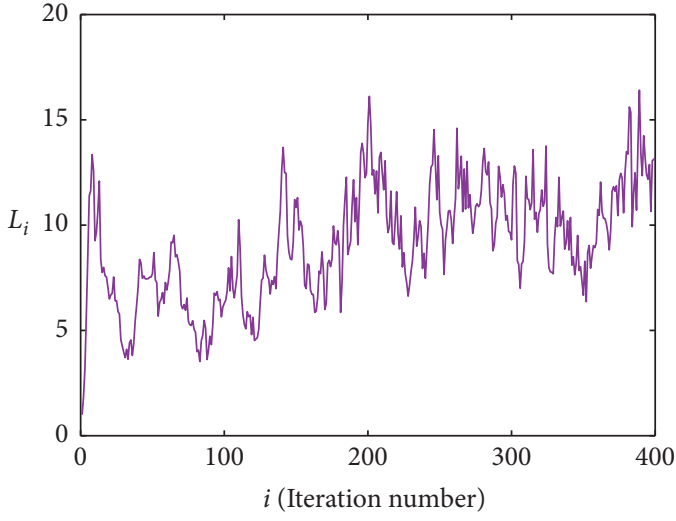

(c)

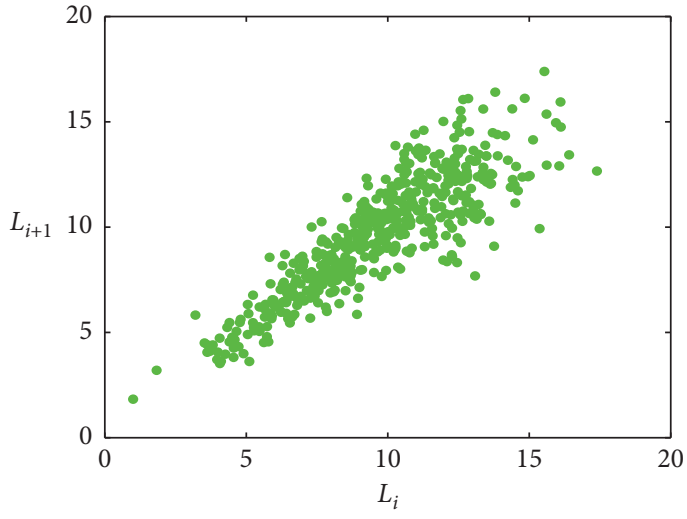

(d)

Figure 8: (a, c) represent $L_{i}$ vs. $i$ graphs with $r=1.5(c=0.5)$ and $3(c=0.5)$, respectively. The corresponding Poincare' plots are given in (b) and (d), respectively. To construct each Poincare plot, we have considered last 5000 points of $L_{i}, i=1,2, \ldots, 50000$.

So, complex dynamics in both systems (1) and (6) are identified successfully over the feasible parametric region under the variable noise strength. However, it does not reflect complexity in the corresponding long-term dynamics of the systems. A quantification is thus needed to measure the complexity of the respective asymptotic dynamics. In the following section, the complexity of (1) and (6) are investigated by weighted recurrence entropy [40].

\section{Recurrence-Based Complexity Analysis}

The weighted recurrence entropy (WRE) is proposed by utilizing Shannon entropy based on the weighted recurrence of phase space. An weighted recurrence for a given $n$-dimensional phase space $P=\left\{x_{i} \in R^{n}\right\}$ is denoted by $w(i, j)$ and defined as

$$
w(i, j)=e^{r(i, j)}, \quad i, j=1,2, \ldots, N,
$$

where $N$ being the length of the trajectory of the phase space and $r(i, j)$ is given by $r(i, j)=-\left\|x_{i}-x_{j}\right\|$. As difference between $x_{i}, x_{j}$ indicates separation between two points, it implies $r(i, j)$ can quantify dispersion between the trajectories. Hence, disorder in $P$ can be measured by $[r(i, j)]$ matrix plot. Furthermore, $w(i, j)$ can measure exponential decay of $r(i, j)$. So, values of $w(i, j)$ always lies between 0 and 1 . It indicates disorder in the phase space can be sampled within $[0,1]$. Then, weight recurrence entropy $\left(S_{w}\right)$ is defined as

$$
S_{w}=-\sum_{s_{k} \in S} p\left(s_{k}\right) \log p\left(s_{k}\right)
$$

where $p\left(s_{k}\right)$ denotes probability of $s_{k} \in S=$ $\left\{s_{k}: s_{k}=1 / N \sum_{j=1}^{T} \omega_{k j}, 1 \leq k \leq T\right\} \quad(T$ being number of events).

We investigate disorder and complexity for both systems (1) and (6), respectively. Only two $[r(i, j)]$ matrix plots are shown in Figures 9(a) and 9(c) for system (1) with $r=2.5$ and 3 , respectively.

From both the figures, it can be observed that greater variation in Figure 9(c) exists compare to the same in
Figure 9(a). It indicates disorder in the phase space of (1) for $r=3$ is greater than that for $r=2.5$. In this way, disorder and hence complexity in the phase space of (1) for the entire range of $r$ can be recognized. To quantify complexity, we further calculate the probabilities $p\left(s_{k}\right)$ using $w(i, j)$ for $r \in[1.5,3]$. Figures 9(b) and 9(d) represent probability curves of the respective disorder given in Figures 9(a) and 9(c). In Figure 9(b), only two sharp peaks can be observed. It signifies that there exists only two variations in Figure 9(a). On the contrary, large number of probabilities in Figure 9(d) indicates that the corresponding phase space is more complex than for $r=2.5$. To verify this, we have calculated respective $S_{w}$ using (8). It has been seen that $S_{w / r=3}=3.821>S_{w / r=1.5}=0.1$. It confirms existence of higher complexity in (1) with $r=3$ than that in same for $r=1.5$. We also investigated complexity in (1) for the entire range of $r$. To do this, fluctuation in $S_{w}$ is measured over $r \in[1.5,3]$. The corresponding curves are shown in Figure 9(e). From the figure, it can be observed that a strong correlation exists between $S_{w}$ and the $K_{c}$ (given in Figure $4(\mathrm{~d})$ ).

Similar analysis is carried out for system (6) with the variation of $r \in[1.5,3]$ and $c \in[0.1,1]$. Figures 10(a) and 10(c) show corresponding $[r(i, j)]$ matrix plots with $r=1.5,3(c=0.5)$, respectively.

From Figures 10(a) and 10(c), similar kind of patterns can be observed in the respective $[r(i, j)]$ matrix, except the values of $r(i, j)$. From the color bar, it can be seen that range of $r(i, j) \in[-2,0]$ for $r=1.5$, whether $r(i, j) \in[-4,0]$ for $r=3$. It indicates greater dispersion between the trajectories for $r=3$ compared to the same for $r=1.5$. It signifies more disordered structures in the corresponding phase space with $r=3$ can be observed compare to the other. In order to verify this, we further calculate respective probability distribution, which is given in Figures 10(b) and 10(d). The distribution for $r=3$ shows variation in $p\left(s_{k}\right)$ for larger range compare to the same for $r=1.5$. Finally, using (8), we have found that $S_{w / r=1.5}=3.4201<S_{w / r=3}=3.58$. It confirms that complexity in (6) for $r=3$ is greater than that for $r=1.5$. Since difference $\Delta S_{w}=S_{w / r=3}-S_{w / r=3}=0.1599$, it corresponds difference between the disorder in the phase 


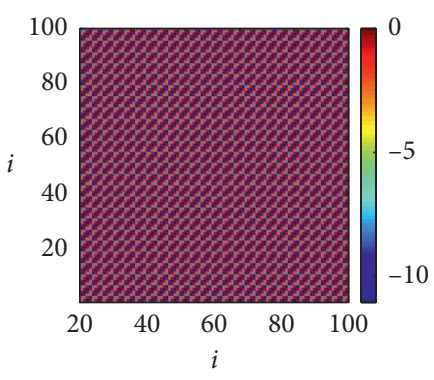

(a)

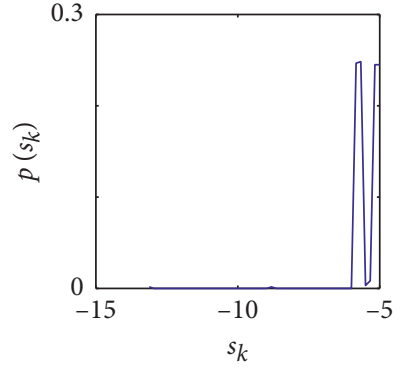

(b)

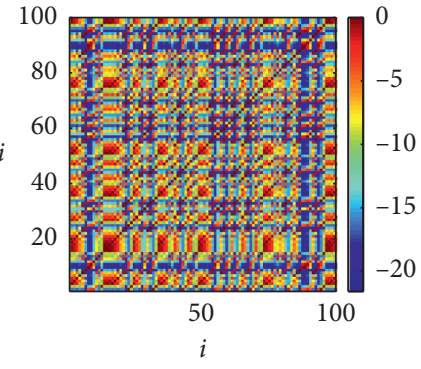

(c)

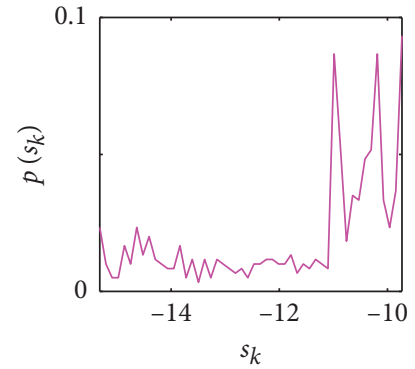

(d)

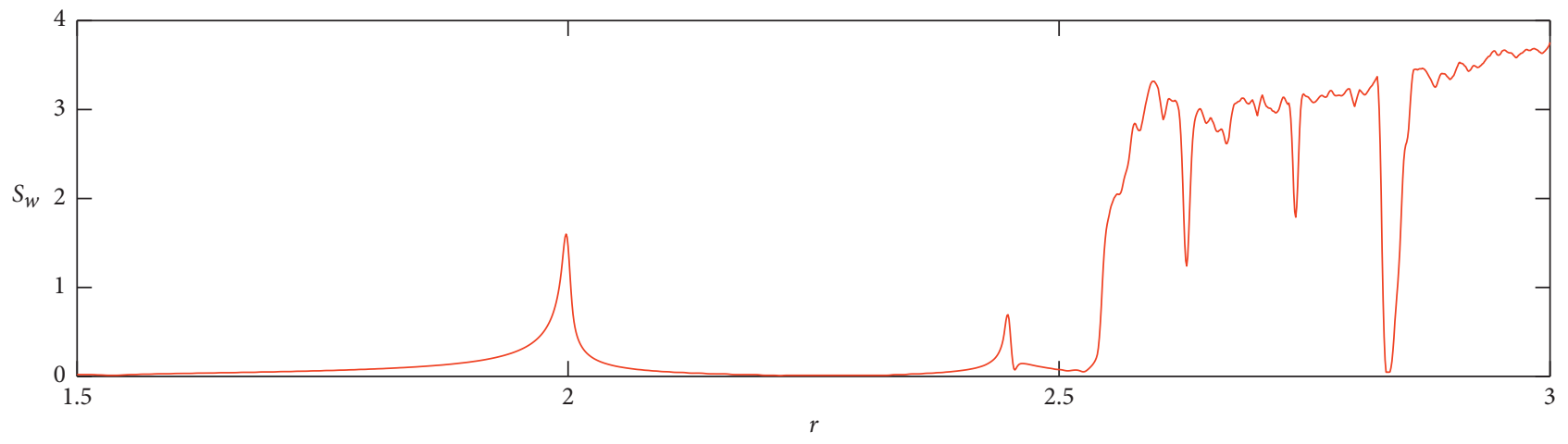

(e)

FiguRE 9: (a, c) $[r(i, j)]$-matrix plot of (1) with $r=2.5$ and 3 respectively. Here, $r(i, j)=-\left\|x_{i}-x_{j}\right\|$. The color bars indicate values of $r(i, j)$ for $i, j=1,2, \ldots, 100$. The corresponding $P\left(s_{k}\right)$ vs. $s_{k}$ plots are given in (b) and (d), respectively. In each case, probabilities are calculated by counting 50 bins. (e) Represents $S_{w}$ vs. $r$ graph of (1) with $r \in[1.5,3]$. To calculate $S_{w}$ by (8), the probabilities are calculated by the aforementioned bin.

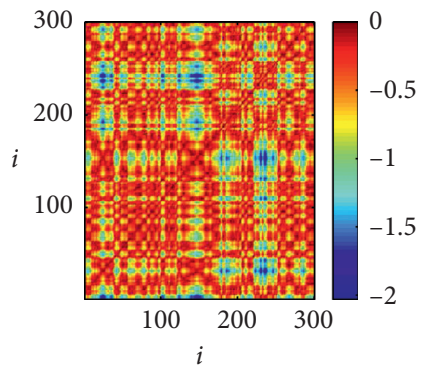

(a)

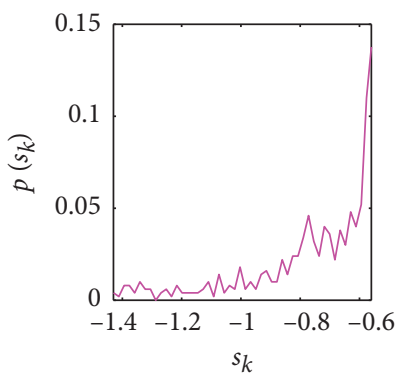

(b)

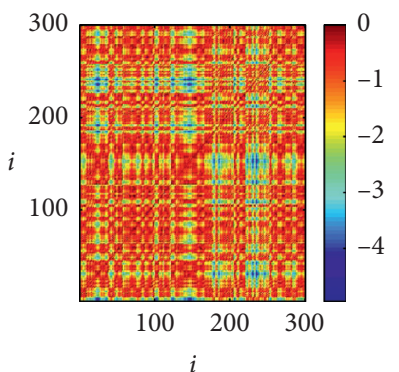

(c)

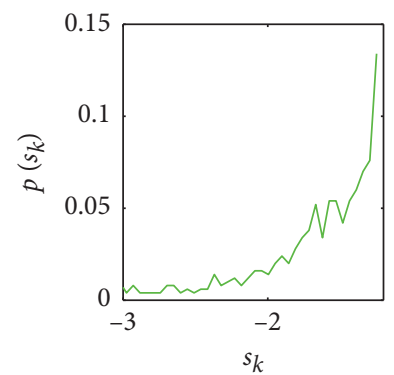

(d)

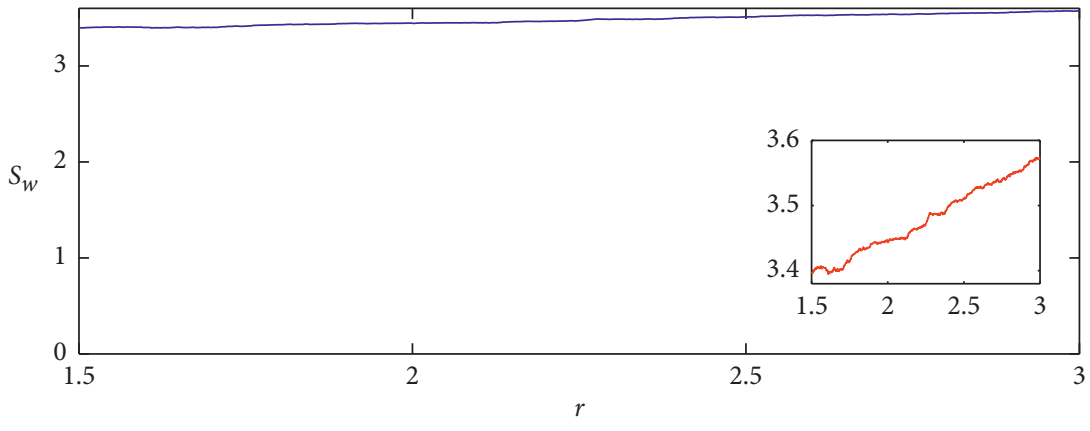

(e)

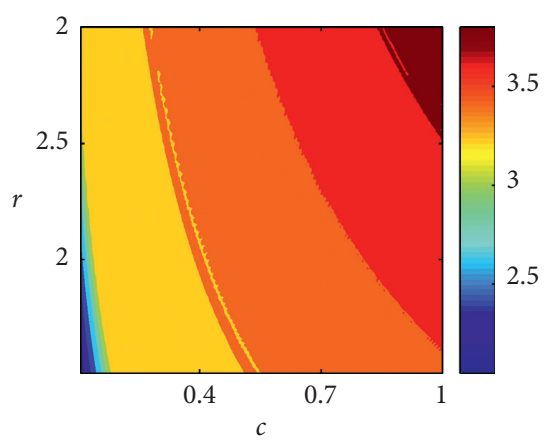

(f)

FIGURE 10: $(\mathrm{a}, \mathrm{c})[r(i, j)]$-matrix plot of (6) with $r=1.5$ and 3 (fixed $c=0.5$ ), respectively. Here, $r(i, j)=-\left\|x_{i}-x_{j}\right\|$. The color bars indicate values of $r(i, j)$ for $i, j=1,2, \ldots, 100$. The corresponding $P\left(s_{k}\right)$ vs. $s_{k}$ plots are given in $(\mathrm{b}, \mathrm{d})$, respectively. In each case, probabilities are calculated by counting 50 bins. (e) $S_{w}$ vs. $r$ graph of (1) with $r \in[1.5,3]$. To calculate $S_{w}$ by (8), the probabilities are calculated by aforementioned bin, keeping fixed $c=0.5$. (f) $\left[S_{w}(r, c)\right]$ matrix plots with $(r, c) \in[1.5,3] \times[0.1,1]$. The associated color bar indicates values of $S_{w}$. 
spaces are very small. Furthermore, complexity in (6) is studied over $r \in[1.5,3]$ with $c=0.5$. Figure $10(\mathrm{e})$ shows the corresponding $S_{w}$ vs. $r$ graphs. It is observed that there exists a strong correlation between the trend in $S_{w}$ and $K_{c}$. It is noted that system (6) can possesses higher complexity compare to the same in (1) at the nonchaotic state.

Finally, we have investigated variation in $S_{w}$ over $(r, c) \in[1.5,3] \times[0.1,1]$. The corresponding contour is shown in Figure 10(f). From the figure, it is observed that values of $S_{w} \in[2,3]$ can be obtained for a very small range of $(r, c)$. In fact, values of $S_{w}$ lies in $(2,4]$ for almost all region.

\section{Conclusions}

In this article, dynamics and complexity are explored for a CBLL and its noise-induced system. The dynamics of the CBLL system have been investigated by equilibrium and its stability, bifurcation, Lyapunov, and 0-1 test analysis. Bifurcation analysis shows periodic as well as multiperiodic solution can be obtained with the variation of intrinsic growth parameter $r$. It has been also observed that multiperiodicity increases with an increase of $r$. It initially indicates the existence of complex dynamics in the CBLL. To verify this, Lyapunov analysis has been performed under the same variation in $r$. Our investigation confirms that the CBLL can have chaotic as well as multiperiodic features. As some of the positive $\lambda$ have been obtained very close to zero, we have carried out 0-1 test analysis. It successfully quantified the chaotic paradigm for the same $r$. Later on, oscillation in language growth and the phase space behaviour have been investigated. It assures that the CBLL system can possess unstable oscillations in $L_{i}$ and hence chaotic dynamics with the various $r$. For the noise-induced CBLL, the 0-1 test method is applied. The effect of noise strength and the variation of $r$ both have been investigated in the noise-induced system. It assures that chaos can be enhanced in the CBLL under the effect of power noise. It has been remarked that deterministic SBLL may contain zero level language growth in some chaotic state. It is not feasible in any real-world cognitive learning phenomena. On the contrary, noise-induced chaotic language growth does not vanish at any condition. It indicates the effectiveness of noise-induced chaos in cognitive language learning. The complexity of both the systems has been quantified using WRE. The corresponding analysis shows that complexity in the CBLL increases with an increase of $r$. On computing complexity, it has been notified that the noise-induced CBLL can produce highly complex phenomena even if its corresponding dynamics was nonchaotic. This phenomenon does not exist for the deterministic case.

\section{Data Availability}

All the data used in this study are included within the text.

\section{Conflicts of Interest}

The authors declare that they have no conflicts of interest.

\section{References}

[1] W. G. Mitchener and M. A. Nowak, "Chaos and language," Proceedings of the Royal Society of London. Series B: Biological Sciences, vol. 271, no. 1540, pp. 701-704, 2004.

[2] S. J. Greenhill, C.-H. Wu, X. Hua, M. Dunn, S. C. Levinson, and R. D. Gray, "Evolutionary dynamics of language systems," Proceedings of the National Academy of Sciences, vol. 114, no. 42, pp. E8822-E8829, 2017.

[3] M. Arbib, "How the brain got language," The Mirror-System Hypothesis, Oxford University Press, Oxford, UK, 2012.

[4] A. Bergs and G. Diewald, Constructions and Language Change, Mouton de Gruyter, Berlin, Germany, 2008.

[5] S. Pinker, The Language Instinct, William Morrow and Company, New York, NY, USA, 1990.

[6] R. Jackendoff, Foundations of Language, Oxford University Press, Oxford, UK, 2002.

[7] V. Barrès and J. Lee, "Template construction grammar: From visual scene description to language comprehension and agrammatism," Neuroinformatics, vol. 12, no. 1, pp. 181-208, 2013.

[8] M. A. Nowak, N. L. Komarova, and P. Niyogi, "Evolution of universal grammar," Science, vol. 291, no. 5501, pp. 114-118, 2001.

[9] P. V. Geert, "A dynamic systems model of cognitive and language growth," Psychological Review, vol. 98, no. 1, pp. 3-53, 1991.

[10] L. Steels and E. Szathmáry, "The evolutionary dynamics of language," BioSystems, vol. 164, pp. 128-137, 2018.

[11] D. L. Freeman, On the Complementarity of Chaos/Complexity Theory and Dynamic Systems Theory in Understanding the Second Language Acquisition Process, Cambridge University Press, Cambridge, UK, 2007.

[12] J. Dong, "A dynamic systems theory approach to development of listening strategy use and listening performance," System, vol. 63, pp. 149-165, 2016.

[13] I. Bashkirtseva, E. Ekaterinchuk, and L. Ryashko, "Stochastic sensitivity analysis and noise-induced chaos in 2D logistictype model," International Journal of Bifurcation and Chaos, vol. 26, no. 4, Article ID 1650053, 2016.

[14] H. Kantz and T. Schreiber, Nonlinear Time Series Analysis, Cambridge University Press, Cambridge, UK, 2nd edition, 2004.

[15] J.-P. Eckmann and D. Ruelle, "Ergodic theory of chaos and strange attractors," Reviews of Modern Physics, vol. 57, pp. 617-656, 1986.

[16] S. Banerjee and M. R. K. Ariffin, "Noise induced synchronization of time-delayed semiconductor lasers and authentication based asymmetric encryption," Optics \& Laser Technology, vol. 45, pp. 435-442, 2013.

[17] T. M. Hoang, S. K. Palit, S. Mukherjee, and S. Banerjee, "Synchronization and secure communication in time delayed semiconductor laser systems," Optik, vol. 127, no. 22, pp. 10930-10947, 2016.

[18] S. K. Palit, N. A. A. Fataf, M. R. Md Said, S. Mukherjee, and S. Banerjee, "Complexity in synchronized and non-synchronized states: A comparative analysis and application," The European Physical Journal Special Topics, vol. 226, no. 10, pp. 2219-2234, 2017.

[19] T. S. Dang, S. K. Palit, S. Mukherjee, T. M. Hoang, and S. Banerjee, "Complexity and synchronization in stochastic chaotic systems," The European Physical Journal Special Topics, vol. 225, no. 1, pp. 159-170, 2016.

[20] T. Schreiber, "Interdisciplinary application of nonlinear time series methods," Physics Reports, vol. 308, no. 1, pp. 1-64, 1999. 
[21] T. S. Parker and L. O. Chua, Practical Numerical Algorithms for Chaotic Systems, Springer, Berlin, Germany, 1989.

[22] G. A. Gottwald and I. Melbourne, "A new test for chaos in deterministic systems," Proceedings of the Royal Society of London. Series A: Mathematical, Physical and Engineering Sciences, vol. 460, no. 2042, pp. 603-611, 2004.

[23] G. A. Gottwald and I. Melbourne, "Comment on "Reliability of the 0-1 test for chaos," Physical Review E, vol. 77, no. 2, Article ID 028201, 2008.

[24] G. A. Gottwald and I. Melbourne, "Testing for chaos in deterministic systems with noise," Physica D: Nonlinear Phenomena, vol. 212, no. 1-2, pp. 100-110, 2005.

[25] F. Franchini, A. R. Its, and V. E. Korepin, "Re'nyi entropy of the XY spin chain," Journal of Physics A: Mathematical and Theoretical, vol. 41, no. 2, p. 025302, 2008.

[26] Y. G. Sinai, "On the notion of entropy of a dynamical system," Dokl Akad Nauk SSSR, vol. 124, pp. 768-771, 1959.

[27] A. Kolmogorov, "Entropy per unit time as a metric invariant of automorphism," Dokl Akad Nauk SSSR, vol. 124, pp. 754-755, 1959.

[28] S. M. Pincus, "Approximate entropy as a measure of system complexity," Proceedings of the National Academy of Sciences, vol. 88, no. 6, pp. 2297-2301, 1991.

[29] J. S. Richman, D. E. Lake, and J. R. Moorman, "Sample entropy," Methods in Enzymology, Academic Press, Cambridge, MA, USA, pp. 172-184, 2004.

[30] S. He and S. Banerjee, "Multicavity formations and complexity modulation in a hyperchaotic discrete system," Physica A: Statistical Mechanics and its Applications, vol. 490, pp. 366-377, 2018.

[31] S. He, S. Banerjee, and B. Yan, "Chaos and symbol complexity in a conformable fractional-order memcapacitor system," Complexity, vol. 2018, pp. 1-15, 2018.

[32] B. Yan, S. Mukherjee, and S. He, "A study on dynamical complexity of noise induced blood flow," The European Physical Journal Special Topics, vol. 228, no. 12, pp. 27692777, 2019.

[33] B. Yan, S. K. Palit, S. Mukherjee, and S. Banerjee, "Signature of complexity in time-frequency domain," Physica A: Statistical Mechanics and its Applications, vol. 535, p. 122433, 2019.

[34] S. Mukherjee, S. Banerjee, and L. Rondoni, "Dispersive graded entropy on computing dynamical complexity," Physica A: Statistical Mechanics and its Applications, vol. 508, pp. 131140, 2018.

[35] S. Banerjee, S. K. Palit, S. Mukherjee, M. R. K. Ariffin, and L. Rondoni, "Complexity in congestive heart failure: A timefrequency approach," Chaos: An Interdisciplinary Journal of Nonlinear Science, vol. 26, no. 3, Article ID 033105, 2016.

[36] S. He, N. A. A. Fataf, S. Banerjee, and K. Sun, "Complexity in the muscular blood vessel model with variable fractional derivative and external disturbances," Physica A: Statistical Mechanics and its Applications, vol. 526, Article ID 120904, 2019.

[37] S. He, S. Banerjee, and K. Sun, "Complex dynamics and multiple coexisting attractors in a fractional-order microscopic chemical system," The European Physical Journal Special Topics, vol. 228, no. 1, pp. 195-207, 2019.

[38] J.-P. Eckmann, S. O. Kamphorst, and D. Ruelle, "Recurrence plots of dynamical systems," Europhysics Letters (EPL), vol. 4, no. 9, pp. 973-977, 1987.
[39] N. Marwan, C. Romano, M. Thiel, and J. Kurths, "Recurrence plots for the analysis of complex systems," Physics Reports, vol. 438, no. 5-6, pp. 237-329, 2007.

[40] D. Eroglu, T. K. D. M. Peron, N. Marwan et al., "Entropy of weighted recurrence plots," Physical Review E, vol. 90, Article ID 042919, 2014. 